\title{
Esophageal Involvement in Pemphigoid: Clinical and Roentgen Manifestations
}

\author{
Farooq P. Agha and Mohammad R. Raji \\ Department of Radiology, West Virginia University Hospital and Medical Center, Morgantown, West Virginia, USA
}

\begin{abstract}
Esophageal involvement in pemphigoid is rare. Review of 32 cases of pemphigoid seen at West Virginia University Hospital between January 1970 and December 1980 revealed 3 patients with esophageal involvement. These cases and review of reported cases of bullous and mucous membrane pemphigoid showed distinct radiographic features of esophageal involvement. These were diffuse inflammatory changes, adhesions, transverse and circumferential webs, and various sizes of strictures. Symptoms of dysphagia may precede radiographic findings by several months or years.
\end{abstract}

Key words: Esophagus inflammation - Esophagus, stricture and web - Pemphigoid, esophageal involvement.

Pemphigoid was first differentiated from pemphigus vulgaris in 1953 [1] by the discovery that its bullae were subepidermal and that acantholysis was absent; it also differs in its clinical features and natural history.

Between January 1970 and December 1980, 32 cases with pemphigoid were seen at the West Virginia University Hospital. All patients had the diagnosis established on the basis of clinical features and histopathological criteria. Twelve patients had bullous pemphigoid and 20 mucous membrane pemphigoid. Three patients had symptoms of dysphagia. Difficulty in swallowing preceded radiographic findings by several months to years. Benign and progressive development of transverse and circumferential webs and strictures led to periodic esophageal dilatations for symptomatic relief. "Esophageal jet phenomenon" was seen with greater frequency as the narrowing of the

Address reprint requests to: Dr. Farooq P. Agha, Department of Radiology, University Hospital, University of Michigan, Ann Arbor, MI 48109, USA lumen of the esophagus progressed. Description of clinical and roentgen features of pemphigoid with particular reference to rare involvement of the esophagus and review of pertinent literature forms the basis of this report.

\section{Case Reports}

\section{Case 1}

This 72-year-old white woman with a 12-year history of benign mucous membrane pemphigoid was initially diganosed in 1968 when she presented with several months' history of recurrent blisters in the throat associated with pain and eventual expelling of fragments of these blisters. She had a few lesions on her scalp. Biopsies of the scalp and oral lesions established the diagnosis of mucous membrane pemphigoid.

Chest and abdominal radiograph was unremarkable except for cholelithiasis. Barium studies of the upper gastrointestinal tract showed no abnormalities at that time. Direct laryngoscopy revealed whitish yellow vericoid lesions in the left side of the epiglottis and vallecula.

She was placed on oral prednisone $5 \mathrm{mg}$ t.i.d. She was already receiving antihypertensive medications.

In September 1969 when she returned to the outpatient clinic, her pharyngeal lesions showed no improvement. She also complained of dysphagia. This difficulty in swallowing became progres sively worse. She was grinding her solid food and medicinal pills before swallowing. The esophagogram at this time was normal. From 1970 to 1980 she had periodic esophagograms (Fig. 1) for progressive dysphagia and required several dilatations of the esophageal stenotic lesions.

Her disease for the most part remained confined to the pharynx, larynx, and esophagus with periods of remission and exacerbations.

Recently she had developed a conjunctival lesion. When last seen in July 1980 the esophageal stricture had improved a little and she had less dysphagia.

\section{Case 2}

This 76-year-old white woman complaining of recurrent ulcers in the mouth for the previous 3 months presented in the outpatient clinic in April 1976. Her past history consisted of an appendectomy, Billroth II gastric surgery for peptic ulcer disease, and rheumatic 
heart disease. She had rheumatoid arthritis for several years for which she took aspirins. She was also on digoxin (Lanoxin ${ }^{\circledR}$ ) and furosemide (Lasix $\left.{ }^{\circledR}\right)$ for chronic congestive heart failure. Physical examination revealed an obese female in no acute distress, however, showing signs of chronic congestive heart failure.

The oral examination revealed two ulcers in the mouth with surrounding tense areas of erythema on the buccal mucosa of the left anterior tonsillar pillar and left side of the base of the tongue. Biopsy of one of these lesions established the diagnosis of benign mucous membrane pemphigoid. She was treated with oral prednisone with periods of remission and exacerbation. In August 1977 she complained of dysphagia of 3 months' duration. An esophagogram was normal. The dysphagia was progressive. In April 1978 the esophagogram (Fig. 2) showed two long strictures in the upper thoracic esophagus. Since then she has had several esophageal dilatations and esophagograms. When the patient was last seen in September 1980, the dysphagia had considerably improved and she had no ulcers in her mouth.

\section{Case 3}

This 81 -year-old man presented in 1980 with recurrent ulcers in his mouth and sore gums for the preceding 1 year. The lesions in the mouth varied in intensity and were very painful at times. Due to these ulcers the patient had lost about 15 pounds over the last 3 months and had even been unable to brush his teeth.

Past history consisted of orchiectomy for hydrocele in 1945 and Billroth II gastrojejunostomy for peptic ulcer disease in 1974.

Physical examination revealed vesicular mucosal lesions in the buccal mucosa. Biopsy of these lesions confirmed the diagnosis of benign mucous membrane pemphigoid. He had no eye or skin lesions.

Recently he had complained of progressive dysphagia. On detailed interrogation, he revealed that symptoms of dysphagia had been present for several months and had recently become worse. An esophagogram (Fig. 3) revealed stricture of the cervical esophagus. No adhesions, webs, or ulceration was seen. Endoscopy confirmed the presence of esophageal stricture. The patient underwent esophageal dilatation with marked improvement of dysphagia.

\section{Clinical Features}

Bullous pemphigoid is one of the chronic generalized bullous dermatoses. It is primarily a disease of the elderly showing no sexual, racial, or ethnic predilection. Clinically, bullous pemphigoid is manifested by tense and usually ungrouped cutaneous bullae arising on a normal, erythematous, or utricarial base. Nikolsky sign is generally negative. The disease shows a predilection for flexual areas and is variably pruritic. The mucous membrane involvement is not uncommon. The mucosal bullae are smaller, fewer in number, and less severe. When present, they are confined to the mouth, but it is rare for oral mucosa to be the first site of lesion. When bullae rupture rapid healing occurs as a rule, rarely resulting in scarring. Fresh crops of bullae arise after variable periods of remission.

Rarely lesions may involve the pharynx, larynx and esophagus [1-4]. The course is chronic and usually self-limited.
Benign mucous membrane pemphigoid (cicatricial pemphigoid) is a chronic blistering disease of mucosal epithelium, which was first defined as an entity and separated from pemphigus by Thost in 1911 [5]. It was variously known as "essential shrinkage of the conjunctiva", chronic pemphigus, or ocular pemphigus, until Lever in 1953 popularized the term "benign mucous membrane pemphigoid." It is generally considered a disease of middle age, and is twice as common in women with no racial or ethnic predilection.

The most characteristic clinical feature of benign mucous membrane pemphigoid is the predilection for recurring bullae of the mucous membrane of the eyes and mouth and the areas of skin adjacent to body orifices. Mucosa of the nasal cavity, pharynx, larynx, and genitals may be involved. Esophageal involvement is rare, resulting in recurrent webs and strictures $[2,6-8]$.

Conjunctival involvement is not a prerequisite for diagnosis of benign mucous membrane pemphigoid; however, eventually $75 \%$ of cases show conjunctival lesions [7]. All lesions show a great tendency for scar formation.

\section{Radiological Features}

The first case of benign mucous membrane pemphigoid (BMMP) with esophageal involvement was reported by Adam in 1910 [9]. This was a 55-year-old woman showing complete obstruction of the esophagus just beyond the pharyngeosophageal junction. Attempts at passage of a bougie resulted in perforation of the esophagus and the patient died 3 days later.

Thost in 1911 [5] reported a case of benign mucous membrane pemphigoid in which involvement of the esophagus had begun in the tenth year of the disease. Six years later [10] he described the autopsy findings of that case confirming esophageal involvement. He reported "diffuse shrinkage and folding of the esophageal mucosa. The folding was longitudinal just as seen in conjunctiva, and there were webs caused by growing together of parts that normally touch each other (adhesive webs). In the upper esophagus a firm thick fold was present which had caused a small accessory lumen, the diameter of a pencil to be separated from the greatly narrowed main lumen. No blisters were seen."

In 1931 Imperatori [6] reported a 68-year-old man with BMMP involving the pharynoesophageal junction.

In Lever's classic description [1], only 5 cases of esophageal involvement in pemphigoid were described, to which Benedict has added 4 more $[1,7]$.

The most recent report by Person and Rogers 

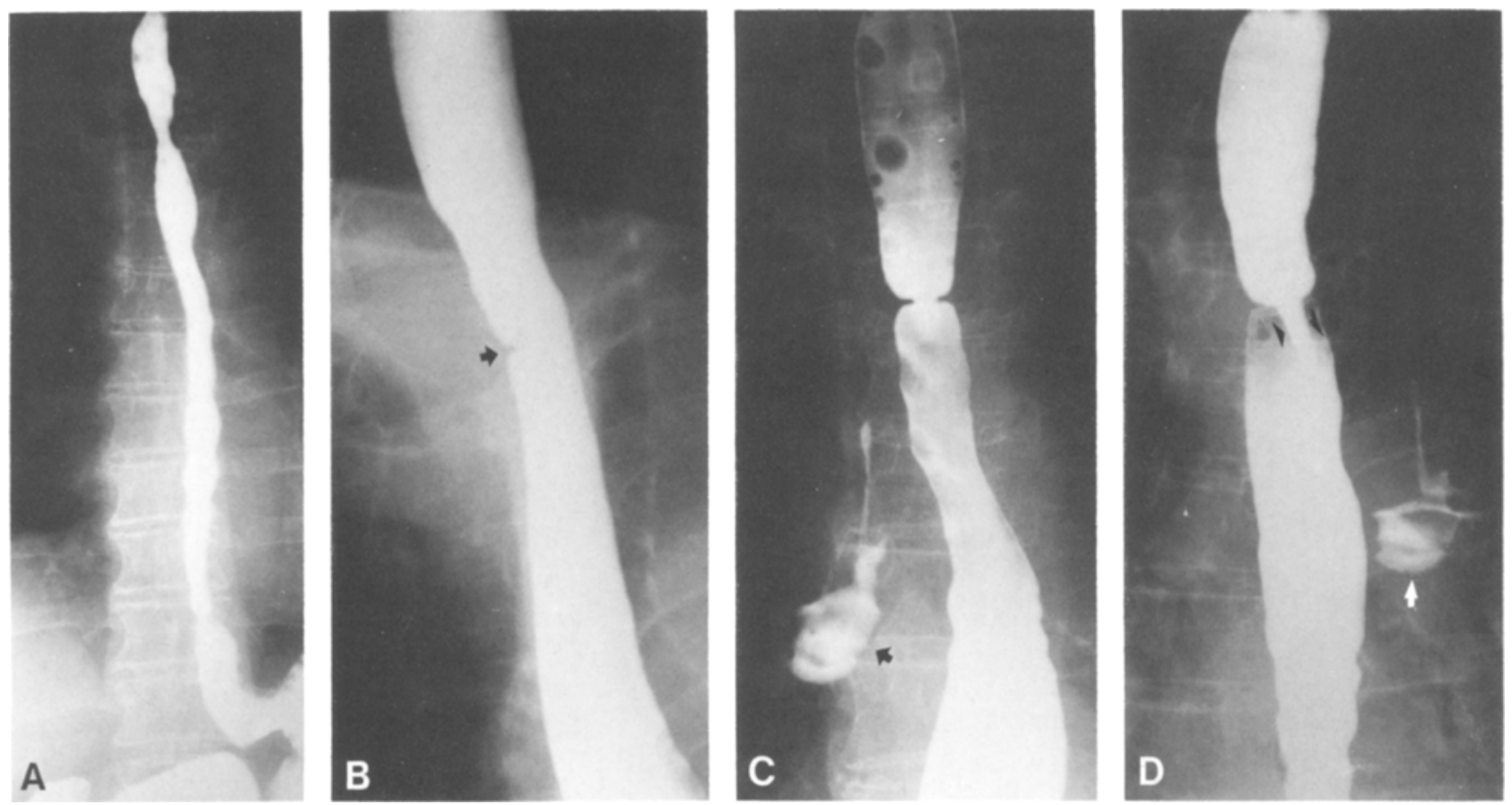

Fig. 1. Case 1. A Esophagogram of August 1970 shows diffuse mucosal inflammation and areas of segmental spasm. B Repeat study in 1972 reveals a small postinflammatory adhesion or web in the upper thoracic esophagus (arrow). C and D Two films from barium evaluation in 1979 demonstrate development of a circumferential web, causing marked narrowing of the lumen and a jet phenomenon during passage of barium (arrowheads in D). Extraluminal barium (arrow) is on the patient's gown
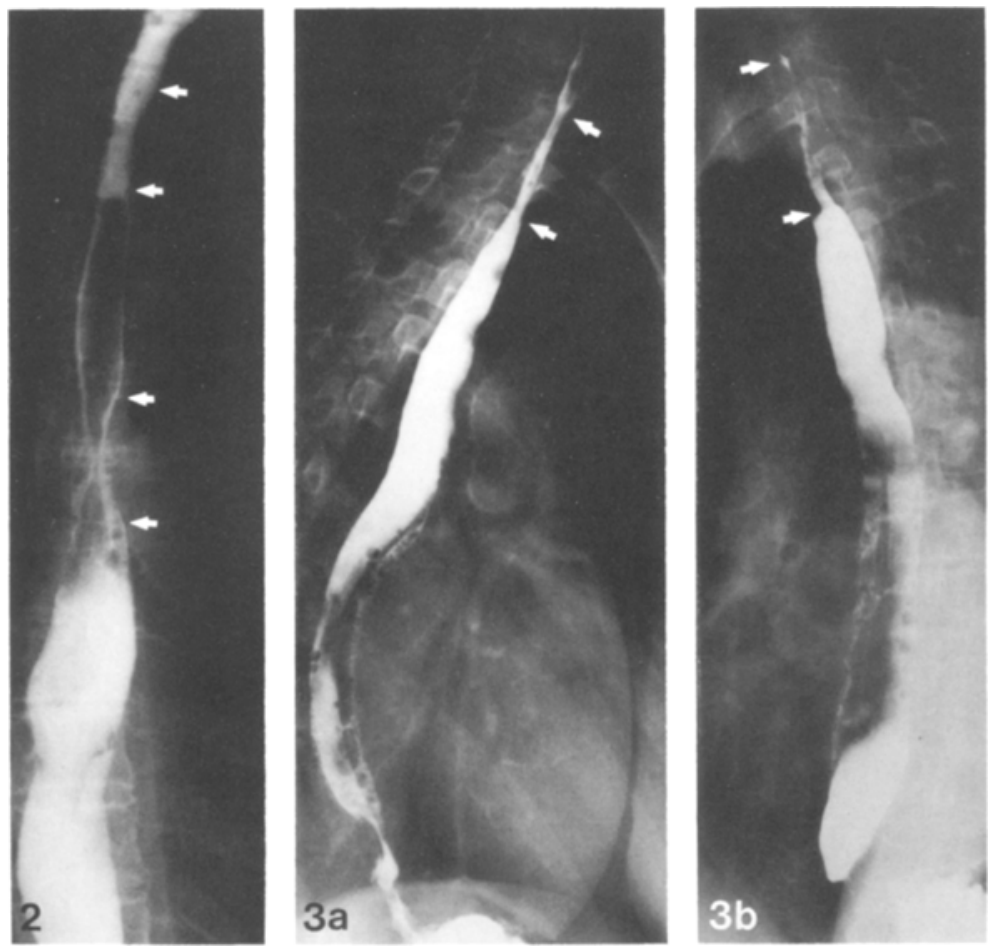

Fig. 2. Case 2. Esophagogram shows two segmental strictures in the proximal esophagus (arrows)

Fig. 3. Case 3. A and $\mathbf{B}$ There is a $5 \mathrm{~cm}$ long stricture in the proximal esophagus (arrows) with a shelving distal border 
[2] from the Mayo Clinic on 84 cases of bullous pemphigoid and 60 cases of mucous membrane pemphigoid revealed esophageal involvement in only $5 \%$ of these cases ( 7 cases: 3 BMMP and 4 bullous pemphigoid).

The symptom of dysphagia usually precedes by several months to several years the radiographic esophageal involvement. The radiological features of esophageal involvement may be variable at different stages of the disease and can be simplified as follows:

\section{Diffuse inflammatory changes}

The early roentgen findings consist of diffuse nonspecific inflammatory changes (Fig. 1 A) manifested as mucosal edema, areas of inconstant narrowing, and spasm. Superficial ulceration secondary to eruption of bullae may be seen.

\section{Adhesions}

As healing progresses, scar formation develops leading to adhesions (Fig. 1 B). These are soft and pliable at first and can be dislodged with the endoscope, but later they become more fibrous and rigid and resist dislodging [7].

\section{Webs}

Postinflammatory webs begin to develop as healing continues, starting as tiny transverse webs (Fig. 1B). As cicatrization progresses the transverse webs by approximation of opposite walls may become circumferential webs (Fig. 1, C-D) with severe dysphagic symptoms. "Esophageal jet phenomenon" usually seen in webs and considered diagnostic of webs [11] was consistently seen in our patients as the circumferential webs progressively narrowed the lumen of the esophagus (Fig. 1D).

\section{Strictures}

Recurrent episodes of disease lead to strictures (Figs. 2 and 3). Strictures may be single, multiple, and of variable lengths. Several strictures may be seen in the same patient (Fig. 2). Once strictures are formed they are usually progressive due to cicatrization.

\section{Complete obstruction of esophageal lumen}

Adam [9] reported total occlusion of the esophageal lumen due to BMMP at $23 \mathrm{~cm}$ from the teeth and a second area of narrowing in the hypopharynx.

Eng et al. [3] reported a case of pemphigoid in which the esophageal mucosa was sloughed and expelled in toto as a hollow cast. There was almost complete regeneration of the mucosa within the next 4 weeks. Early recognition of esophageal involvement in pemphigoid is important because early adhesions can be dislodged and severe stenosis can be prevented. Therefore, any patient with bullous or mucous membrane pemphigoid complaining of dysphagia should have a careful roentgenologic evaluation of the esophagus.

\section{References}

1. Lever WF: Pemphigus. Medicine 32:1-123, 1953

2. Person JR, Rogers R: Bullous and cicatricial pemphigoid. Mayo Clinic Proc 52:54-66, 1977

3. Eng TY, Hogan WJ, Jordan RE: Esophageal involvement in bullous pemphigoid - a possible cause of gastrointestinal hemorrhage. BrJ Dermatol 99:207-210, 1978

4. Sharon P, Green ML, Rachmilewitz D: Esophageal involvement in bullous pemphigoid. Gastrointest Endoscop 24:122-123, 1978

5. Thost A: Der chronische Schleimhaut-Pemphigus der oberen Luftwege. Arch Laryngol Rhinol (Berl) 25:459-478, 1911

6. Imperatori CJ: Stricture of the esophagus caused by pemphigus (BMMP). Ann Otol-Rhinol Laryngol 40:1192-1193, 1931

7. Benedict EB, Lever WF: Stenosis of the esophagus in benign mucous membrane pemphigus. Ann Otol Rhinol 61:1120-1133, 1952

8. Muller $\mathrm{H}$ : Esophageal stenosis in a case of pemphigoid benignum. Laryngol Rhinol Otol 96:282-283, 1977

9. Adam C: Untersuchungen zur Pathologie des Pemphigus conjunctivae. Ztschr Augenheilk 23:35-48, 1910

10. Thost A: Ueber Schleimhaut-Pemphigus. Arch Laryngol Rhinol (Berl) 31:599-604, 1917

11. Shauffer IA, Phillips AE, Sequerik J: "The jet phenomenon" - a manifestation of esophageal webs. AJR 129:747-748, 1977

12. Foroozan P, Enta T, Winship DH. Trier IS : Loss and regurgitation of the esophageal mucous in pemphigoid. Gastroenterology $53: 548-555,1967$

Received: July 24, 1981; accepted: September 11, 1981 